\title{
AURC All Normalized by Weight
}

National Cancer Institute

\section{Source}

National Cancer Institute. AURC All Normalized by Weight. NCI Thesaurus. Code C92345.

The area under the urinary excretion rate curve (AURC) from time zero to the last measurable rate divided by the weight. 